\title{
Electrophysiological Evidence for Hyperfocusing of Spatial Attention in Schizophrenia
}

\author{
Johanna Kreither, ${ }^{1}$ Javier Lopez-Calderon, ${ }^{1,2}$ Carly J. Leonard, ${ }^{1}$ Benjamin M. Robinson, ${ }^{3}$ Abigail Ruffle, ${ }^{3}$ Britta Hahn, ${ }^{3}$ \\ James M. Gold, ${ }^{3}$ and Steven J. Luck ${ }^{1,2}$ \\ ${ }^{1}$ Center for Mind and Brain and ${ }^{2}$ Department of Psychology, University of California, Davis, Davis, California 95616, and ${ }^{3}$ Maryland Psychiatric Research \\ Center, Department of Psychiatry, University of Maryland School of Medicine, Baltimore, Maryland 21201
}

A recently proposed hyperfocusing hypothesis of cognitive dysfunction in schizophrenia proposes that people with schizophrenia (PSZ) tend to concentrate processing resources more narrowly but more intensely than healthy control subjects (HCS). The present study tests a key prediction of this hypothesis, namely, that PSZ will hyperfocus on information presented at the center of gaze. This should lead to greater filtering of peripheral stimuli when the task requires focusing centrally but reduced filtering of central stimuli when the task requires attending broadly in the periphery. These predictions were tested in a double oddball paradigm, in which frequent standard stimuli and rare oddball stimuli were presented at central and peripheral locations while event-related potentials were recorded. Participants were instructed to discriminate between the standard and oddball stimuli at either the central location or at the peripheral locations. PSZ and HCS showed opposite patterns of spatial bias at the level of early sensory processing, as assessed with the P1 component: PSZ exhibited stronger sensory suppression of peripheral stimuli when the task required attending narrowly to the central location, whereas HCS exhibited stronger sensory suppression of central stimuli when the task required attending broadly to the peripheral locations. Moreover, PSZ exhibited a stronger stimulus categorization response than HCS, as assessed with the P3b component, for central stimuli when the task required attending to the peripheral region. These results provide strong evidence of hyperfocusing in PSZ, which may provide a unified mechanistic account of multiple aspects of cognitive dysfunction in schizophrenia.

Key words: attention; event-related potential; hyperfocusing; p300; schizophrenia

\section{Significance Statement}

Schizophrenia clearly involves impaired attention, but attention is complex, and delineating the precise nature of attentional dysfunction in schizophrenia has been difficult. The present study tests a new hyperfocusing hypothesis, which proposes that people with schizophrenia (PSZ) tend to concentrate processing resources more intensely but more narrowly than healthy control subjects (HCS). Using electrophysiological measures of sensory and cognitive processing, we found that PSZ were actually superior to HCS in focusing attention at the point of gaze and filtering out peripheral distractors when the task required a narrow focusing of attention. This finding of superior filtering in PSZ supports the hyperfocusing hypothesis, which may provide the mechanism underlying a broad range of cognitive impairments in schizophrenia.

\section{Introduction}

Recent research suggests that several aspects of cognitive dysfunction in schizophrenia can be explained by a tendency of people with schizophrenia (PSZ) to concentrate processing resources

Received 0ct. 16, 2016; revised Jan. 25, 2017; accepted Feb. 10, 2017.

Author contributions: J.K., J.L.-C., C.J.L., B.M.R., B.H., J.M.G., and S.J.L. designed research; J.K., B.M.R., and A.R. performed research; J.K. and S.J.L. analyzed data; J.K., C.J.L., B.M.R., B.H., J.M.G., and S.J.L. wrote the paper.

This study was supported by NIH Grant R01 MH065034 (J.M.G., S.J.L.).

The authors declare no competing financial interests.

Correspondence should be addressed to Steven J. Luck, Center for Mind and Brain, University of California, Davis,

267 Cousteau Place, Davis, CA 95618. E-mail: sjluck@ucdavis.edu.

DOI:10.1523/JNEUROSCI.3221-16.2017

Copyright $\odot 2017$ the authors $\quad 0270-6474 / 17 / 373813-11 \$ 15.00 / 0$ more intensely but more narrowly than healthy control subjects (HCS) (Luck et al., 2014). This hyperfocusing hypothesis can explain reduced visual working memory capacity in PSZ relative to HCS (Leonard et al., 2012), larger spatial and temporal cuing effects (Hahn et al., 2012a,b; Spencer et al., 2011), greater capture of attention by distractors that partially match task goals (Mayer et al., 2012; Luck et al., 2014; Sawaki et al., 2017), a narrower span of attention during visual search (Elahipanah et al., 2010, 2011), and impairments in the ability of PSZ to distribute attention broadly (Hahn et al., 2012a; Gray et al., 2014). Thus, hyperfocusing may be a fundamental characteristic of schizophrenia that underlies many of the specific deficits in cognitive performance that have been observed in this disease. 
$90 \%$ blue and $10 \%$ yellow (or vice versa) at each location

Attend inner or attend outer (separate blocks)

Press to indicate yellow or blue in attended region; withhold response for unattended region

\section{A Example Stimulus Sequence}

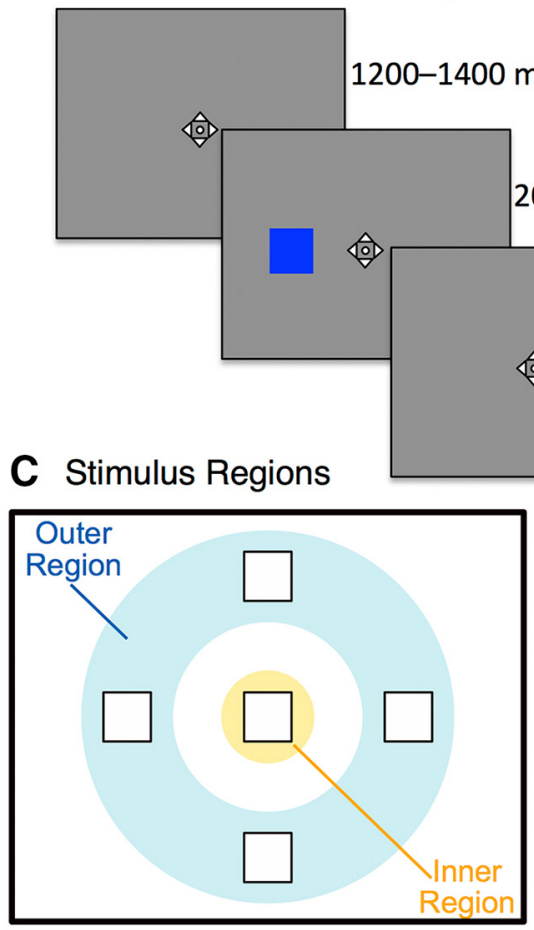

D Useful Field of View (UFOV) Task
B Attentional Reminder

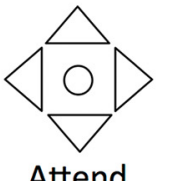

Attend

outer

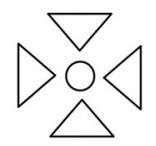

or

Attend inner

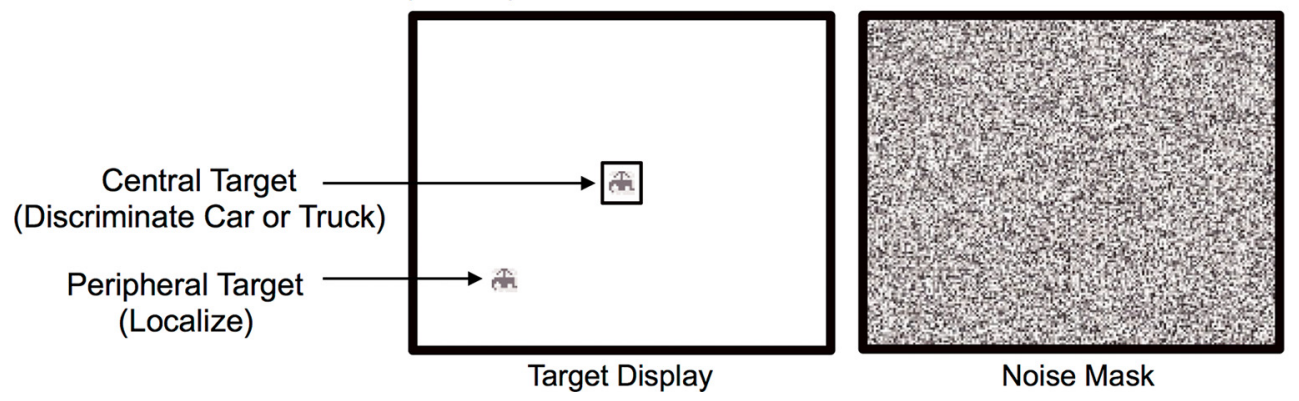

Figure 1. A, Example stimulus sequence from the double oddball task. The stimuli were colored squares ( $90 \%$ blue and $10 \%$ yellow or vice versa) presented at the central location on $50 \%$ of trials and at one of the four peripheral locations on the remaining $50 \%$. Participants attended to the inner region in some trial blocks and to the outer region in other trial blocks. They were instructed to press one button for blue stimuli in the attended region, to press another button for yellow stimuli in the attended region, and to make no response for stimuli in the unattended region. $\boldsymbol{B}$, Enlarged view of the attentional reminder stimulus that was present in the middle of the display to ensure that participants knew at all times which region should be attended. $C$, Diagram of the inner and outer stimulus regions. $\boldsymbol{D}$, Example stimuli from the divided attention condition of the UFOV task. The target display was presented for a given period of time and then immediately followed by the noise mask. Participants reported whether the central stimulus was a car or truck and then clicked on one of eight locations on the screen to report the position of the peripheral target. The duration of the target display was adjusted with an adaptive staircase to determine the duration that would lead to an accuracy level of $75 \%$ correct.

Most of the evidence for hyperfocusing was derived from behavioral measures that reflect the combined impact of several processing systems. The goal of the present study was to test the hyperfocusing hypothesis using electrophysiological measures that reflect specific processing systems. We used a variant of a double oddball paradigm used previously to study attention deficit hyperactivity disorder (López et al., 2006). The task (Fig. 1) involved either attending narrowly to the foveal region or attending broadly to a set of four peripheral locations. Within each of these two regions, $90 \%$ of the stimuli were standards of one color and $10 \%$ were oddballs of a different color. Participants were instructed to attend either to the narrow inner region or to the broad outer region and discriminate between the standards and oddballs in the instructed region, ignoring all stimuli in the other region.

The center of gaze has a natural competitive advantage for attention (Fischer and Weber, 1993), and the tendency of PSZ to hyperfocus was predicted to lead to an exaggeration of this tendency in the double oddball task. Previous research shows that healthy individuals detect parafoveal targets more rapidly than peripheral targets in visual search tasks, even when controlling for cortical magnification (Woodman and Luck, 2003), and this 
Table 1. Group demographics (mean \pm SD)

\begin{tabular}{|c|c|c|}
\hline & PSZ & $\mathrm{HCS}$ \\
\hline Age & $34.4 \pm 10.2$ (range, $19-56$ ) & $35.2 \pm 10.5$ (range, $18-57)$ \\
\hline Male/female & $23: 8$ & $19 / 8$ \\
\hline $\mathrm{AA} / \mathrm{A} / \mathrm{C} / \mathrm{M}$ & $12 / 1 / 17 / 1$ & $10 / 1 / 15 / 1$ \\
\hline Education (years) & $13.2 \pm 2.2$ & $15.2 \pm 2.0^{* *}$ \\
\hline Parental education $^{a}$ & $14.0 \pm 2.6$ & $14.0 \pm 2.1$ \\
\hline WASI IQ & $99.9 \pm 16.4^{*}$ & $111.2 \pm 10.2^{*}$ \\
\hline MCCB total score & $36.2 \pm 12.7^{* *}$ & $50.3 \pm 8.9^{* * b}$ \\
\hline BPRS total score & $32.6 \pm 8.7$ (range, 21-54) & \\
\hline SANS total score & $25.0 \pm 13.0$ (range, $10-43$ ) & \\
\hline LOFS total score & $20.9 \pm 5.0(\text { range, } 13-29)^{b}$ & \\
\hline
\end{tabular}

AA, African American; A, Asian; C, Caucasian; M, mixed race; LOFS, Level Of Functioning Scale (Hawk et al., 1975). ${ }^{a}$ Average over mother's and father's years of education.

${ }^{b}$ Data are unavailable for one subject.

${ }^{*} p<0.05 ;{ }^{* *} p<0.001$ (significant difference between PSZ and HCS in independent sample $t$ test).

effect is exaggerated in PSZ (Elahipanah et al., 2010). Similarly, PSZ make more hypometric saccades than HCS, consistent with an exaggerated attentional weight for the center of gaze (Leonard et al., 2013; Luck et al., 2014).

To assess the neural mechanisms of hyperfocusing, we recorded event-related potentials (ERPs) and focused on the P1 component, which reflects extrastriate sensory processing, and the $\mathrm{P} 3 \mathrm{~b}$ component, which reflects the allocation of higher-level processing resources (Di Russo et al., 2003; Polich, 2012). We predicted that PSZ would exhibit an enhanced ability (relative to HCS) to focus narrowly on the central location and suppress the $\mathrm{P} 1$ and P3b components for to-be-ignored peripheral stimuli. By contrast, we predicted that PSZ would exhibit an impaired ability (relative to HCS) to focus broadly on the peripheral region and suppress the P1 and P3b components for to-be-ignored central stimuli.

Participants also received a standardized behavioral assessment of the ability to distribute attention broadly, the useful field of view (UFOV) task (Ball and Owsley, 1993). This task assesses how well individuals can divide their attention between simultaneously presented central and peripheral stimuli, and performance predicts real-world outcomes such as automobile accidents (Myers et al., 2000). We have previously shown that PSZ are impaired in this task and that the degree of impairment is correlated with reductions in working memory capacity (Gray et al., 2014). We predicted that the electrophysiological measures of central hyperfocusing in the double oddball task would be associated with the degree of impairment on the UFOV task, thus providing the first steps toward a mechanistic link between the neural activity and behavior impairments.

\section{Materials and Methods}

Participants. The participants consisted of 34 people with schizophrenia $(N=25)$ or schizoaffective disorder $(N=9)$ and $27 \mathrm{HCS}$ (see gender and other demographic information in Table 1). As described below, 3 PSZ had excessive artifacts and were excluded from all analyses, leaving a final sample size of 31 PSZ.

Diagnoses were established using a best estimate approach, combining material from past medical records, collateral informants (when available), and the criteria from the Diagnostic and Statistical Manual of Mental Disorders, fourth edition (DSM-IV; American Psychiatric Association) as established from the results of the Structured Clinical Interview for DSM-IV-TR Axis I disorders (First et al., 1997). Final diagnosis was reached at a consensus conference involving clinical staff and chaired by one of the authors (J.M.G). All PSZ were clinically stable outpatients who had been receiving the same medications, at the same dose, for at least 4 weeks before study participation. Three PSZ were receiving typical antipsychotics, 29 were receiving atypical antipsychotics, and 2 were receiving both. Additional medications in PSZ included antidepressants $(N=15)$, mood stabilizers $(N=6)$, anxiolytics $(N=8)$, sleep medications $(N=2)$, and antiparkinson medication $(N=4)$.

HCS were recruited by Internet advertising and random digit dialing in the greater Baltimore metropolitan area. They were screened using the Structured Clinical Interview for DSM-IV Axis I disorders and Axis II personality disorders, which revealed no current diagnosis of any Axis I disorder or Axis II schizophrenia spectrum disorder. In addition, HCS self-reported no lifetime history of psychosis and no psychotic disorders in first-degree relatives. The participants in both groups were free of other medical or neurologic disorders that might interfere with test performance, including substance abuse or dependence within the last 12 months.

Several symptom measures were obtained, including the Scale for the Assessment of Negative Symptoms (SANS; Andreasen, 1984) and the Brief Psychiatric Rating Scale (BPRS; Overall and Gorham, 1962). Demographic information, neuropsychological test scores, and psychiatric ratings are provided in Table 1 . No significant group differences were found in age $\left(t_{(56)}=0.53, p>0.5\right)$, race $\left(\chi^{2}=0.31, p>0.9\right)$, $\operatorname{sex}\left(\chi^{2}=\right.$ $0.106, p>0.7)$, or parental education $\left(t_{(56)}=0.36, p>0.7\right)$. The PSZ had completed fewer years of education than the HCS $\left(t_{(56)}=3.44\right.$, $p=0.001)$, which presumably reflects the effect of schizophrenia on educational attainment. We also found significant reductions in overall cognitive ability in PSZ relative to HCS in the Wechsler Abbreviated Scale of Intelligence (WASI IQ; Wechsler, 1999; $t_{(56)}=-3.112, p=0.003$ ) and the MATRICS Consensus Cognitive Battery (MCCB; Nuechterlein et al., 2008; total, $\left.t_{(56)}=-4.815, p<0.001\right)$. These impairments in overall cognitive ability were comparable to those typically observed in studies of stable outpatients (Johnson et al., 2013).

Double oddball task. As illustrated in Figure 1, participants were instructed to attend either to an inner region or to an outer region and to perform an oddball discrimination task in the attended region (López et al., 2006). This task provides ERP measures of two distinct processing mechanisms involved in attention. The $\mathrm{P} 1$ component is a sensoryevoked response that is generated in extrastriate visual cortex (Di Russo et al., 2003). If attention is strongly focused on one spatial region, P1 amplitude is typically reduced for stimuli presented in other regions, reflecting an early sensory filtering process (Hillyard et al., 1998). This effect appears to reflect a filtering of unattended locations rather than an enhancement of attended locations (Luck et al., 1994; Luck and Hillyard, 1995; Luck, 1995).

The P3b component is a well-validated index of the allocation of higher-level cognitive processing resources (Polich, 2012). P3b amplitude is much larger for rare oddball stimuli than for frequent standard stimuli when the stimuli are actively discriminated. By contrast, little or no difference in P3b amplitude is observed between oddballs and standards if they are not actively attended to. P3b amplitude scales with the amount of higher-level processing resources devoted to the eliciting stimulus (Isreal et al., 1980), thus providing a measure of the extent to which participants allocate attention to a to-be-ignored stimulus. Note that many distinct neurocognitive processes are active during the period of the $\mathrm{P} 3 \mathrm{~b}$ component, but by focusing on the difference in amplitude between the oddball and standard stimuli, most other processes are subtracted out (Potts et al., 2002; Luck, 2014).

The stimuli were presented on an LCD monitor with a $60 \mathrm{~Hz}$ refresh rate and a gray background at a viewing distance of $100 \mathrm{~cm}$. The monitor delay was measured from the display with a photodiode, and the data were shifted with respect to the delay interval (which was $30 \mathrm{~ms}$ ). As shown in Figure $1 A$, a white fixation point and attentional reminder were continuously present at the center of the screen (except when occluded by an oddball task stimulus). The attentional reminder $\left(0.6^{\circ}\right.$ of visual angle; Fig. $1 B$ ) consisted of four triangles pointing inward (in the attendinner condition) or outward (in the attend-outer condition).

The stimuli for the double oddball task consisted of a sequence of blue or yellow squares $\left(2.5^{\circ}\right)$. As shown in Figure $1 C$, each stimulus was presented for $200 \mathrm{~ms}$ either at the fixation point (inner region; $p=0.5$ ) or at one of four locations centered $4.5^{\circ}$ above, below, to the left, or to the right of the fixation point (outer region; $p=0.125$ for each of the four loca- 
tions). In half of the trial blocks, $90 \%$ of the stimuli in a given region were blue and $10 \%$ were yellow, and this was reversed for the other trial blocks. Stimulus order was independently randomized within each region. Each stimulus was followed by an interstimulus interval of 1200-1400 ms (rectangular distribution). Because only one stimulus was presented at a time, we could independently assess ERPs and behavioral responses to stimuli in the inner and outer regions.

Participants were instructed to attend to the central location in attendinner blocks and to all four peripheral locations in attend-outer blocks. They were required to respond on a game pad, using the dominant hand, for every stimulus presented in the attended region, making an indexfinger response for yellow and a middle-finger response for blue, or vice versa, counterbalanced across participants. The mapping of colors to fingers stayed the same across trial blocks for a given participant, independent of which color was rare or frequent. The participants were instructed to make no response when the stimulus appeared in the unattended region.

The recording session was divided into four trial blocks, each consisting of 320 stimuli. Each block was divided into four miniblocks, separated by short breaks, and a longer break was provided after each full block. Each full block was one of the four combinations of attend inner versus outer and yellow rare versus blue rare, and the blocks occurred in a randomized order. Each participant received a total of 288 standards and 32 oddballs for each combination of stimulus location and direction of attention.

Recording and analysis. The electroencephalogram (EEG) was recorded using a BioSemi ActiveTwo EEG recording system. Thirty-two electrodes were affixed in an elastic cap at a subset of locations from the extended 10/20 System (FP1, FP2, F3, Fz, F4, F7, F8, C3, Cz, C4, P1, P2, P3, Pz, P4, P5, P6, P7, P8, P9, P10, T7, T8, PO3, POz, PO4, PO7, PO8, O1, $\mathrm{Oz}, \mathrm{O}_{2}$, INz). To detect eye movements and blinks, the electrooculogram was recorded from electrodes placed lateral to the outer canthi and below the left eye. The single-ended voltage was recorded between each electrode site and a common mode sense electrode. The signals were lowpass filtered with a fifth-order sinc filter (half power cutoff at $208 \mathrm{~Hz}$ ) and digitized at $1024 \mathrm{~Hz}$.

Data processing was performed in MATLAB with the open-source EEGLAB (Delorme and Makeig, 2004) and ERPLAB (Lopez-Calderon and Luck, 2014) toolboxes. The data were filtered off-line using a noncausal Butterworth bandpass filter (half-amplitude cutoffs at 0.1 and 30 $\mathrm{Hz}, 24 \mathrm{~dB}$ /octave roll-off). All scalp electrodes were referenced off-line to the average of the left and right mastoids. To correct for blink artifacts, independent component analysis was applied to the continuous data after the deletion of sections containing extreme values, and a single component reflecting blinks was identified in each participant on the basis of timing and scalp topography and then removed (Jung et al., 2000). Standard artifact rejection procedures (Luck, 2014) were also applied to eliminate epochs containing horizontal eye movements, miscellaneous artifactual voltage deflections, and blinks that occurred within $200 \mathrm{~ms}$ of stimulus onset and may have therefore prevented perception of the stimulus (measured in a channel that was not subjected to artifact correction). In our studies of patient populations, we always exclude any participants for whom $>50 \%$ of trials were rejected because of artifacts; three PSZ were rejected for this reason in the present study. In the remaining participants, only $4.7 \%$ of trials were rejected because of artifacts. Trials were also excluded if the behavioral response was incorrect. Of the 32 oddball trials that were tested in a given subject for each condition, an average of 30.4 trials (and a minimum of 23 trials) contributed to the averaged ERP waveform.

Averaged ERP waveforms were computed for the standard and oddball stimuli in each attentional condition, averaging over which color was rare, using a $200 \mathrm{~ms}$ prestimulus interval. To maximize the signal-tonoise ratio, the average waveforms for targets and for standards were collapsed across the electrodes within an a priori region of interest (the $\mathrm{O} 1, \mathrm{Oz}$, and $\mathrm{O}_{2}$ electrode sites). $\mathrm{P} 1$ amplitude was quantified as the mean voltage from 100 to $220 \mathrm{~ms}$. This measurement window was determined by using a collapsed localizer approach in which a waveform is created that is averaged across all conditions and groups, providing an unbiased means of defining the measurement window (Brooks et al.,
2017; Luck and Gaspelin, 2017). In the present case, we began by creating a grand average attended-minus-unattended difference wave, averaged across all conditions, for each group. We then normalized these grand averages to eliminate any overall differences in amplitude between PSZ and HCS, and then we averaged these two grand averages together into a collapsed grand average. We then found the time points at which the amplitude was $20 \%$ of the maximum voltage of the collapsed grand average, and we used these points to define the time window for measuring the amplitude of the P1 attention effect (100-220 ms).

To isolate the $\mathrm{P} 3 \mathrm{~b}$ probability effect, $\mathrm{P} 3 \mathrm{~b}$ amplitude was measured from rare-minus-frequent difference waves (Potts et al., 2002; Luck et al., 2009) after averaging the waveforms across an a priori region of interest (the P1, Pz, and P2 electrode sites). P3b amplitude was quantified as the positive area between 370 and $770 \mathrm{~ms}$. Positive area is defined as the area of positive voltage bounded by the waveform and the zero voltage line. This method minimizes the impact of temporally adjacent components of opposite polarity (e.g., the N2 wave; Luck, 2014). However, because the value can never be negative, chance is not $0 \mu \mathrm{V}$; consequently, permutation testing (using the method of Sawaki et al., 2012) was used in analyses that compared the positive area to chance. Again, the measurement window was determined by a collapsed localizer in which the rare-minus-frequent difference waveforms were averaged across all conditions and both groups.

Useful field of view task. Because the double oddball task was not designed to provide a sensitive behavioral measure of attention, each participant was also tested in the UFOV task (Ball and Owsley, 1993; Myers et al., 2000) on a separate day. We used a commercial version of the task (Ball and Roenker, 1998) that includes three separate conditions, tested in separate trial blocks (for details of the stimuli and procedure, see Gray et al., 2014). The key condition for the present study is called the "divided attention" condition. In this condition, a car or truck is presented centrally, and an additional car is presented at a peripheral location (Fig. $1 D)$. The task is to first report whether the central stimulus is a car or truck, and to then report the location of the peripheral target. The stimulus display is terminated by a mask. The stimulus display duration is adjusted by means of an adaptive staircase to find the duration at which the participant is $75 \%$ correct. This threshold value is the dependent variable. For a trial to be considered correct, both the central discrimination and the peripheral localization must be correct. This task differs from the double oddball task insofar as the central and peripheral locations are attended simultaneously in the UFOV task, but it is similar to the attend-outer condition of the double oddball task insofar as it requires distributing attention over a broad region. Consequently, PSZ who exhibit a strong tendency to focus centrally and have difficulty distributing attention broadly in the attend-outer condition in the double oddball task should also perform poorly in the UFOV task.

The UFOV task also includes a processing speed condition, in which only the central target is presented, and a selective attention condition in which peripheral distractors (triangles) are presented along with the peripheral target. These conditions are part of the standard task, but were not relevant for the present study and will not be discussed further.

\section{Results}

\section{Double oddball behavioral performance}

Behavior in the double oddball task was quantified in two ways. First, we asked whether participants responded to stimuli in the to-be-attended region (hit rate) and withheld responses to stimuli in the to-be-ignored region (correct rejection rate), independent of whether the stimulus color was correctly categorized. PSZ tended to have a reduced hit rate for to-be-attended stimuli in the outer region (Fig. 2A) and a reduced correct rejection rate for to-be-ignored stimuli in the inner region (Fig. $2 B$ ), as would be expected if they were biased toward stimuli in the inner region. However, performance was well above $90 \%$ for both measures, and in two-factor ANOVAs, the group by stimulus region interaction did not reach significance for either hit rate $\left(F_{(1,56)}=2.10\right.$, $p=0.15)$ or correct rejection rate $(p=0.11)$. Overall perfor- 

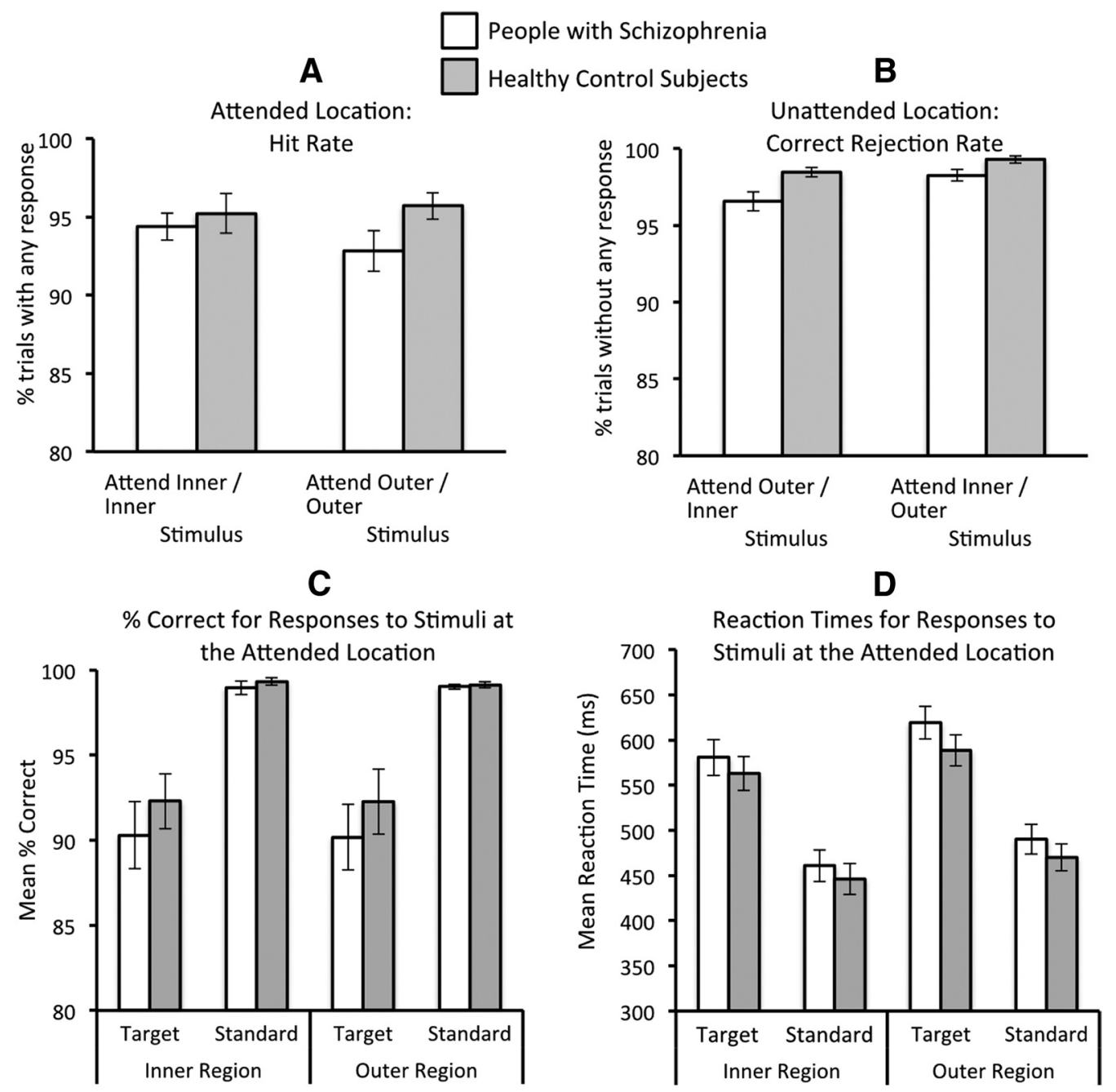

Figure 2. Behavioral performance in the double oddball task. $A, B$, Percentage of trials on which participants responded to inner and outer stimuli in the attended region $(A)$ and the unattended region $(\boldsymbol{B})$, regardless of whether the response was correct for the stimulus color. This reflects the extent to which participants attended to the correct region. $\boldsymbol{C}, \boldsymbol{D}$, Discrimination accuracy for responses to stimuli presented in the attended region $(\boldsymbol{C})$ and the reaction time for correct responses $(\boldsymbol{D})$. Error bars show the SEM.

mance was slightly worse for PSZ than for HCS, but the main effect of group was significant only for the correct rejection rate $\left(F_{(1,56)}=7.24, p=0.009\right)$.

A second set of ANOVAs asked how quickly and accurately participants categorized the color of the stimuli at the attended location, using factors of group, stimulus region (inner vs outer), and stimulus category (rare vs frequent). Color discrimination accuracy was lower (Fig. 2C) and reaction time was slower (D) for the rare targets than for the frequent standards, which led to a significant main effect of stimulus category for both measures $(p<0.001)$. Participants were also slightly faster for stimuli in the inner region than in the outer region, as confirmed by a main effect of stimulus region for reaction time $(p<0.001)$. Although PSZ were slower on average than HCS, the main effect of group was not significant for either reaction time or $\%$ correct ( $p$ values $>0.35$ ), nor were there any significant interactions involving group ( $p$ values $>0.3$ ).

Because the double oddball task was not designed to provide a sensitive measure of attention, the general lack of significant group differences was not surprising and facilitated the interpretation of the electrophysiological results (Kappenman and Luck, 2016): because the two groups displayed high and similar levels of behavioral performance, any differences in electrophysiological responses between PSZ and HCS cannot be attributed to problems in understanding task instructions, poor motivation, etc. Moreover, as will be described in a later section, PSZ did exhibit a significant behavioral impairment in the UFOV task, which is a sensitive and well-validated measure of the ability to distribute attention broadly in the periphery.

\section{Sensory filtering: the $\mathbf{P} 1$ component}

Figure 3, $A$ and $B$, show the grand average waveforms for stimuli presented in the to-be-attended and to-be-ignored regions, averaged across the occipital electrode sites and across the standard and oddball stimuli. In PSZ, the P1 wave for stimuli presented in the outer region was smaller in the attend-inner condition relative to the attend-outer condition, indicating that PSZ successfully filtered the sensory response to the peripheral stimuli when attending the inner region. However, the P1 wave for stimuli presented at the central location was not influenced by which region was attended in PSZ, suggesting that PSZ were unable to filter the sensory response to the central stimuli when attending the outer region. In HCS, the opposite pattern was observed: the P1 wave for stimuli presented at the central location was larger when the inner region was attended than when the outer region 
was attended, but the $\mathrm{P} 1$ wave for stimuli presented in the outer region was not influenced by which region was attended.

To minimize the number of factors in the statistical analysis and thereby decrease the experimentwise type I error rate (Cramer et al., 2016; Luck and Gaspelin, 2017), we collapsed the data into attended-minusignored difference scores for each stimulus region, as illustrated in Figure $3 C$. These difference scores were entered into a two-way ANOVA with factors of group and stimulus region (inner vs outer). The main effects were not significant $(F<1)$, but the group by stimulus region interaction was $\left(F_{(1,56)}=\right.$ $4.21, p=0.04)$. As shown in Figure 3C, PSZ exhibited a large attentional modulation (attended-minus-ignored difference) for the outer region $\left(t_{(30)}=2.91, p=0.007\right)$ but not for the inner region $\left(t_{(30)}=0.058, p>\right.$ 0.9 ), whereas HCS exhibited a large attentional modulation for the inner region $\left.t_{(26)}=2.91, p=0.007\right)$ but not for the outer region $\left(t_{(26)}=0.049, p>0.9\right)$. Thus, PSZ effectively suppressed the peripheral stimuli when attending to the inner region, but they failed to suppress the central stimuli when attending to the outer region. HCS, in contrast, modulate the response to the central stimuli but not to the peripheral stimuli.

\section{Higher-level resource allocation: the P3b component}

Figure 4 shows the grand averages for the rare and frequent stimuli to illustrate the P3b probability effect. As discussed earlier, the main analyses focused on the rareminus-frequent difference waves, which are shown in Figure 5. Whereas the P1 analysis examined the suppression of sensory processing at the to-be-ignored location relative to the to-be-attended location, collapsed across standards and oddballs, the P3b analysis asked how well participants were able to suppress the differential processing of the oddballs relative to the standards at the to-be-ignored location. The hyperfocusing hypothesis predicts that PSZ will devote processing resources to the central region even when this region is task irrelevant, leading to an enhanced $\mathrm{P} 3 \mathrm{~b}$ rare-minus-frequent difference score for to-be-ignored stimuli presented in this region.

Consistent with this prediction, we found that the rareminus-frequent difference waves (averaged over the P1, Pz, and $\mathrm{P} 2$ electrode sites) for to-be-ignored central stimuli were larger in PSZ than in HCS $\left(t_{(56)}=1.98, p=0.05\right)$. In other words, when the task required attending to the outer region, stimuli in the inner region produced a larger $\mathrm{P} 3 \mathrm{~b}$ probability effect in $\mathrm{PSZ}$ than in HCS. However, the P3b probability effect for stimuli in the outer region in the attend-inner condition was approximately the same in PSZ and HCS $\left(t_{(56)}=0.56, p=0.58\right)$. The rare-minusfrequent difference scores were analyzed in a two-way ANOVA with factors of group and stimulus region (inner vs outer). The main effect of stimulus region was significant $\left(F_{(1,56)}=12.60, p<\right.$ $0.001)$, reflecting larger $\mathrm{P} 3 \mathrm{~b}$ effects for to-be-ignored stimuli in
People with Schizophrenia Healthy Control Subjects
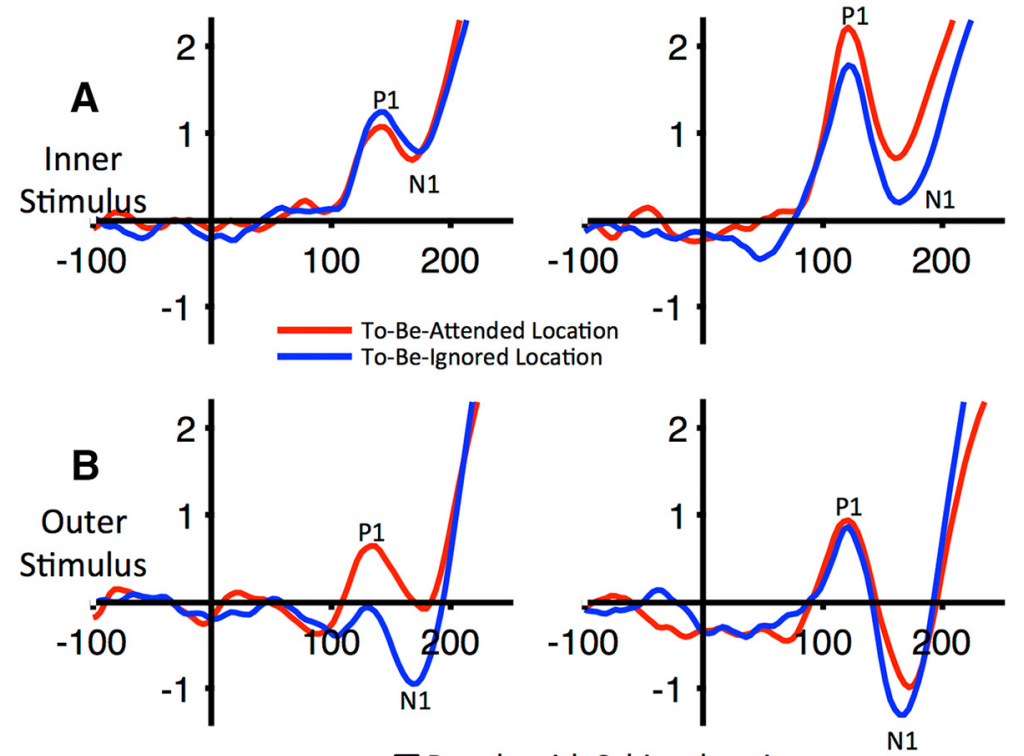

People with Schizophrenia

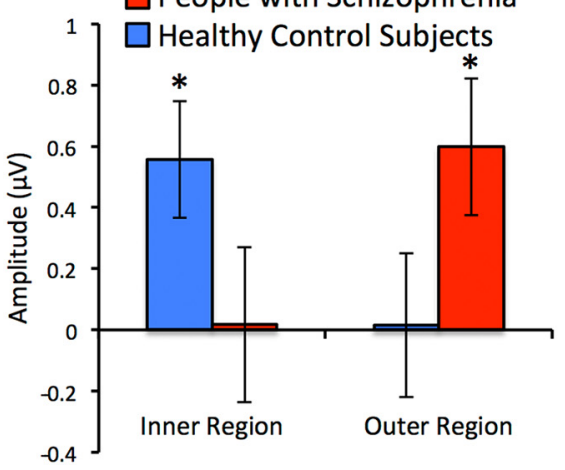

Figure 3. ERP data collapsed across standards and oddballs and the occipital electrode sites $\left(0 z, 01\right.$, and $\left.\mathrm{O}_{2}\right)$ to show the P1 wave for stimuli in the attended and unattended regions. $\boldsymbol{A}$, Waveforms for stimuli in the inner region. $\boldsymbol{B}$, Waveforms for stimuli in the outer region. $\boldsymbol{C}$, Mean P1 amplitudes measured from the waveforms shown in $\boldsymbol{A}$ and $\boldsymbol{B}$. Error bars show the SEM. Asterisks 政 significant suppression of the $P 1$ wave when the outer region was attended to compared to when the inner region was attended to $(A$, right), whereas PSZ did not $(A$, left).

the outer region than for stimuli in the inner region for both groups. The group main effect was not significant $\left(F_{(1,56)}=0.41\right.$, $p=0.52)$, but the group by stimulus region interaction was $\left(F_{(1,56)}=4.10, p=0.05\right)$, reflecting the group difference for the inner but not the outer region (Fig. $5 C$ ). In addition, permutation testing showed that the rare-frequent difference scores for to-beignored stimuli were significantly greater than zero for the inner region in PSZ but not in HCS. Thus, HCS were able to suppress the $\mathrm{P} 3 \mathrm{~b}$ effect for to-be-ignored stimuli in the inner region but PSZ were not.

We did not predict any group differences in the rare-minusfrequent difference scores for the to-be-attended stimuli given that the standard oddball discrimination task was so easy. However, we analyzed the $\mathrm{P} 3 \mathrm{~b}$ wave for to-be-attended stimuli for the sake of completeness, again using rare-minus-frequent difference scores and a two-way ANOVA with factors of group and stimulus region. The difference scores were slightly larger for inner-region stimuli than for outer-region stimuli in both groups, leading to a significant main effect of stimulus region $\left(F_{(1,56)}=6.30\right.$, $p=0.02$ ). However, neither the main effect of group nor the 


\section{People with Schizophrenia}

\section{_... Rare \\ Frequent}

Inner Stimulus Attend Inner

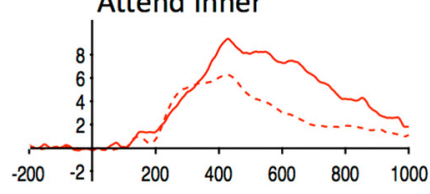

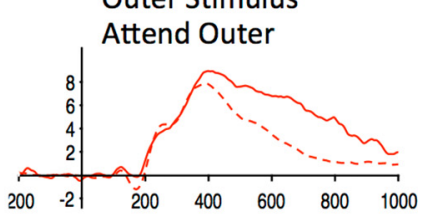

\section{Healthy Control Subjects}

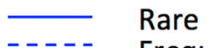

---- Frequent
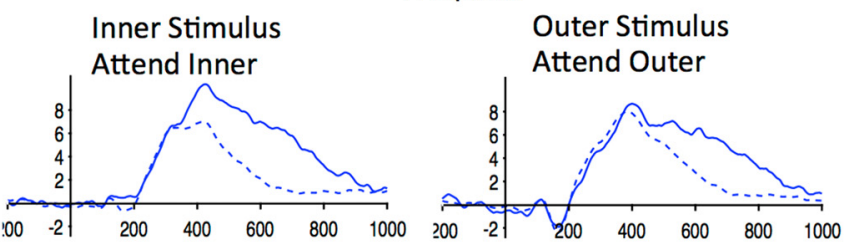
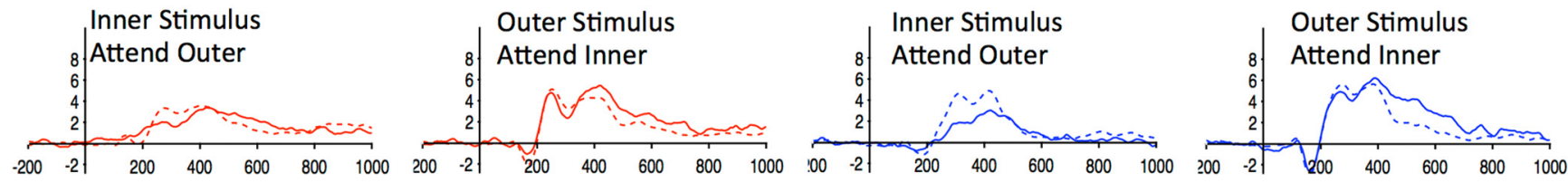

Figure 4. Grand average ERP waveforms, separated for standards and oddballs and collapsed across parietal electrode sites (Pz, P1, and P2) to show the P3b probability effect. $A$, Waveforms for stimuli presented in the attended region. $\boldsymbol{B}$, Waveforms for stimuli presented in the unattended region.

\section{Rare-Minus-Frequent Difference Waves}
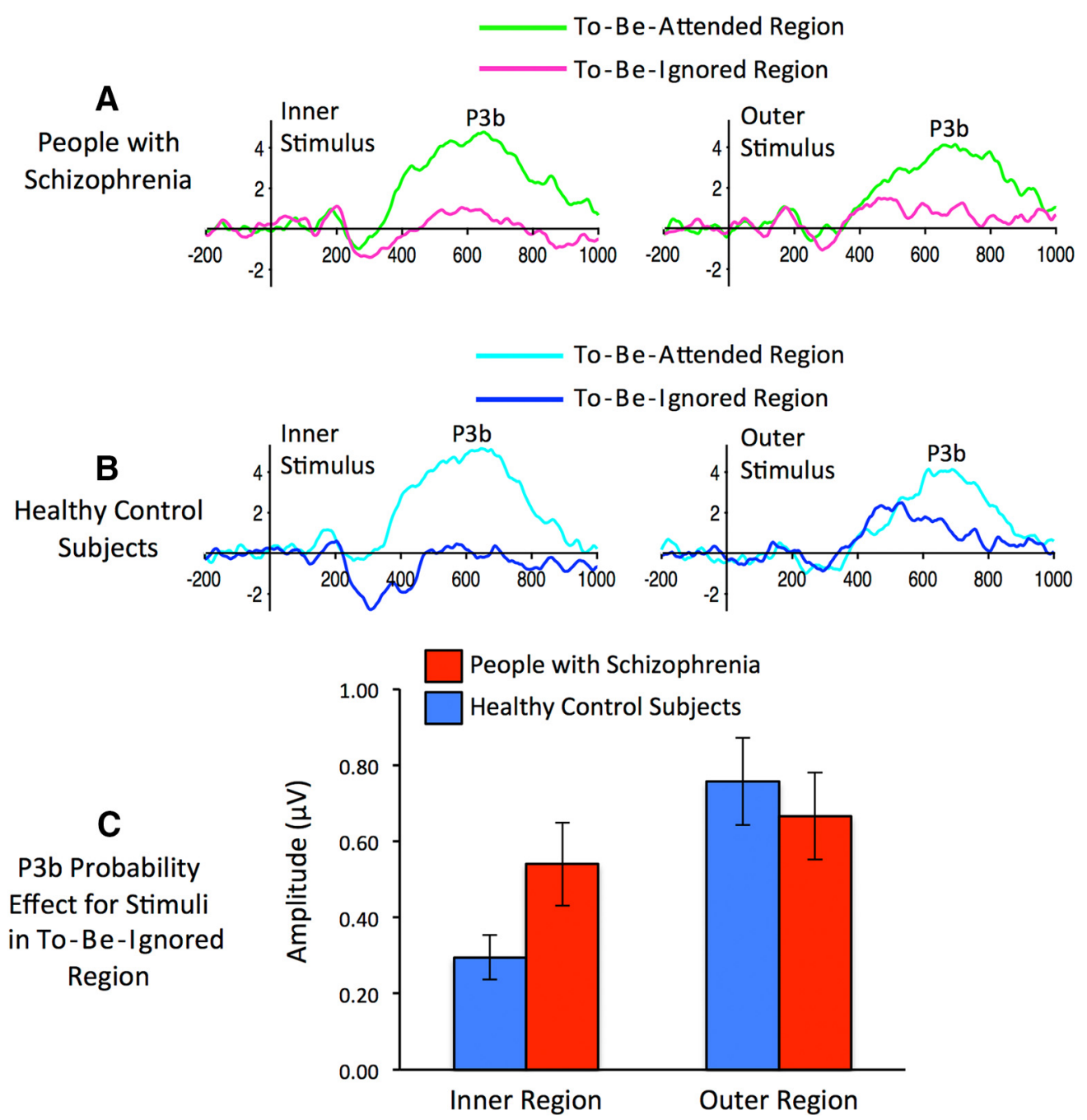

Figure 5. $A-C$, Grand average rare-minus-frequent difference waves collapsed across parietal electrode sites (Pz, P1, and P2) in people with schizophrenia $(A)$ and healthy control subjects $(\boldsymbol{B})$, along with mean rare-minus-frequent difference scores for the to-be-ignored stimuli (C). Error bars show the SEM. 
People with Schizophrenia

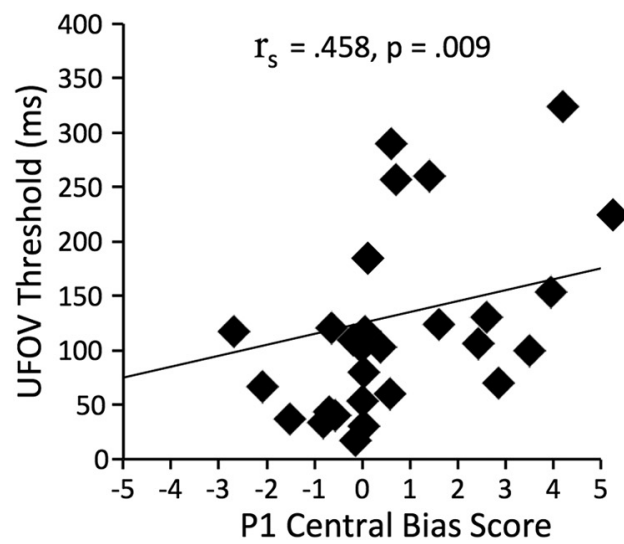

Healthy Control Subjects

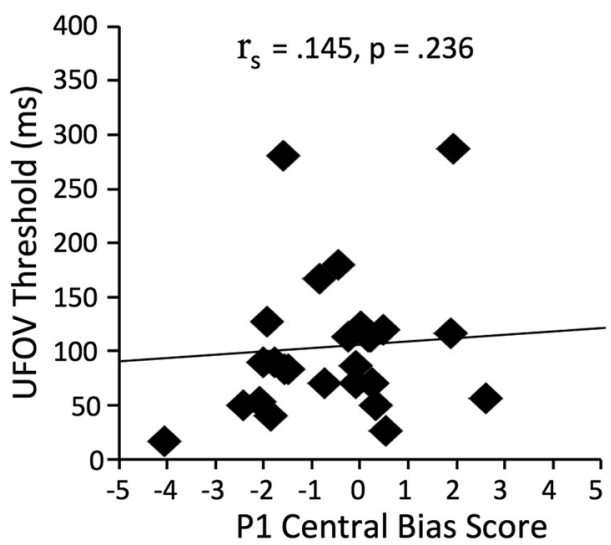

Figure 6. Scatter plot of the relationship between threshold in the UFOV task and the P1 central bias score in people with schizophrenia (left) and healthy control subjects (right). Note that higher thresholds in the UFOV task reflect worse performance.

group by stimulus region interaction was significant ( $F$ values $<1$ ). The absence of a group difference in P3b amplitude for attended stimuli is not unusual: reductions in PSZ relative to HCS are typically more pronounced for auditory than visual stimuli (Jeon and Polich, 2003), and two previous studies found no group difference in rare-minus-frequent difference scores from visual oddball tasks (Potts et al., 2002; Luck et al., 2009).

\section{Medication analyses}

To assess whether the ERP results may reflect medication effects, we tested the correlation between medication dosage (chlorpromazine equivalent, calculated according to Andreasen et al., 2010) and our main dependent variables (P1 attention effect for the inner and outer regions; $\mathrm{P} 3 \mathrm{~b}$ probability effect for the inner and outer to-be-attended regions; P3b probability effect for the inner and outer to-be-ignored regions). There were no significant correlations between dosage and any of these variables (Pearson's $r$ between -0.191 and $0.265, p \geq 0.2$ for all correlations).

\section{Correlations with useful field of view}

The mean display duration threshold in the UFOV task was higher in PSZ (94.1 ms; SEM, 34.0) relative to HCS (35.9 ms; SEM, 9.6). Thresholds were highly skewed and significantly deviated from the normal distribution in both PSZ and HCS according to the Anderson-Darling test, so nonparametric statistics were used for all analyses involving these thresholds. The average threshold was significantly higher in PSZ than in HCS according to a Mann-Whitney $U$ test $(U=305.5, p<0.05)$, replicating a previous finding of impaired UFOV performance in PSZ (Gray et al., 2014).

To assess correlations between the neural measures from the double oddball task and the behavioral UFOV measure while minimizing the total number of correlations, a central bias score was computed for the P1 and P3b components. For P1 amplitude, the central bias score was computed as the attended-minusunattended difference score for the outer region minus the attended-minus-unattended difference score for the inner region. This score reflected the extent to which filtering of unattended stimuli was more effective for the outer region than for the inner region, and hence a bias toward the inner region. For P3b amplitude, the central bias score was computed as the rareminus-frequent difference score for unattended stimuli in the inner region minus the rare-minus-frequent difference score for unattended stimuli in the outer region. This score reflected the extent to which the $\mathrm{P} 3 \mathrm{~b}$ probability effect for unattended stimuli was greater for the inner region than for the outer region, reflecting greater processing of unattended stimuli in the inner region.

We computed the Spearman rho rank-order correlation between each central bias score and the UFOV threshold score separately for PSZ and HCS (Fig. 6, scatter plots). In PSZ, UFOV performance exhibited a significant positive correlation with the P1 central bias score $(r=0.458, p=0.009)$. In other words, PSZ who exhibited more sensory processing bias toward the central region in the double oddball task also exhibited poorer performance (higher thresholds) in the UFOV task. This correlation was not significant in HCS $(r=0.145, p=0.47)$. Thus, in PSZ, our electrophysiological measure of attentional bias toward the point of gaze at the sensory level was associated with impaired performance in a task that requires attention to be distributed broadly. For the P3b component, there was no significant association with UFOV performance in either PSZ $(r=-0.210, p=$ $0.26)$ or HCS $(r=0.060, p=0.77)$.

\section{Discussion}

This study was a direct test of the hyperfocusing hypothesis of schizophrenia, which proposes that PSZ tend to focus processing resources more intensely but more narrowly than HCS. In the spatial domain, this hypothesis predicts an exaggeration of the link between attention and gaze position that has been observed in healthy individuals (Fischer and Weber, 1993). Specifically, we predicted that PSZ would exhibit enhanced attentional processing of information presented at the point of gaze and enhanced filtering of peripheral stimuli, along with an impaired ability to distribute attention broadly to the periphery and ignore information presented at the point of gaze. We found multiple pieces of evidence supporting these predictions.

\section{Major findings}

First, at the stage of the initial sensory response (indexed by the P1 wave; Fig. 3), PSZ were biased to attend to central stimuli over peripheral stimuli. Specifically, PSZ strongly suppressed the sensory response to stimuli in the outer region when attending to the inner region, but they failed to filter the sensory response to stimuli in the inner region when attending to the outer region. 
HCS exhibited the opposite pattern, with stronger attentional filtering in the inner region than the outer region.

Second, the sensory bias toward the central region indexed by the P1 wave in PSZ correlated with a behavioral measure of divided attention derived from the well-validated UFOV task. Specifically, PSZ who exhibited a stronger bias toward the inner region as quantified with the P1 attention effect also exhibited poorer behavioral performance when required to distribute attention broadly in the UFOV task. This correlation is important because the ERP and behavioral measures have contrasting strengths. The P1 measure provides a precise operationalization of the concept of hyperfocusing because it allows us to quantify the extent to which stimuli within a given region can be filtered by spatial attention at the stage of extrastriate visual cortex (Di Russo et al., 2003). Performance in the UFOV task involves a naturalistic situation where attention is spread across both the point of gaze and the periphery; in fact, individual differences in UFOV performance predict important real-world outcomes such as automobile accidents (Myers et al., 2000). Thus, the finding of a significant relationship between the electrophysiological and behavioral measures provides an important step toward a mechanistic account of how neural changes in attentional filtering may be related to real-world performance in PSZ.

The UFOV results were important also because the double oddball task used to measure the ERP effects was not designed to provide a sensitive behavioral measure of the distribution of attention. Indeed, it was designed to be very simple, leading to near-ceiling performance in both groups, which avoids problems that arise in interpreting neural data when patients and controls differ in performance (Kappenman and Luck, 2016). It would be useful for future studies to develop a version of this task that is optimized for behavior rather than for electrophysiology, but the lack of behavioral differences between groups in the present ERPoptimized version does not weaken our conclusions, especially given the significant differences in the UFOV task.

A third major finding was that, at the postperceptual stage indexed by the P3b wave, the probability effect was greater in PSZ than in HCS for to-be-ignored stimuli presented in the inner region (Fig. 5). This indicates that PSZ devote high-level processing resources to stimuli at the center of gaze even when this region is task irrelevant. Interestingly, this is the opposite of the pattern observed in children with attention deficit/hyperactivity disorder, who exhibit an exaggerated P3b probability effect for unattended stimuli in the outer region (López et al., 2006).

In contrast to the P1 component, the exaggerated bias for the P3b component was not correlated with UFOV performance. Additional research will be needed to determine whether this is an artifact of differences in psychometric properties between the P1 and P3b measures or whether it reflects a more fundamental difference between the sensory filtering process reflected by the P1 attention effect and the higher-level cognitive process reflected by the P3b probability effect.

\section{Additional issues}

An unanticipated finding was that HCS appeared to exhibit an enlarged N2 component in the rare-minus-frequent difference wave for the inner stimuli when they were instructed to attend to the outer region (Fig. 5B). We speculate that this may reflect an "unmasking" of the $\mathrm{N} 2$ due to the elimination of the partially overlapping P3b component. Indeed, the N2 component for the inner stimuli was larger in both PSZ and HCS in the attend-outer condition than in the attend-inner condition, which could also be explained by an unmasking of the $\mathrm{N} 2$ component because $\mathrm{P} 3 \mathrm{~b}$ amplitude was substantially reduced when the stimuli were to be ignored.

Another unanticipated finding was that HCS exhibited a P1 attention effect only for the central stimuli and not for the peripheral stimuli. We speculate that this may reflect differences in perceptual load between the attend-inner and attend-outer conditions (for review, see Lavie, 2010). Specifically, perceptual load for HCS is presumably quite low in the attend-inner condition, which may decrease the need to filter the peripheral distractors (Lavie, 1995). Attending peripherally is presumably more demanding, leading HCS to filter the central stimuli when instructed to attend peripherally. Although PSZ should also experience low perceptual load when instructed to attend centrally, the mechanisms that produce hyperfocusing may lead to filtering of peripheral distractors nonetheless.

An important aspect of the present findings is that whereas most studies of schizophrenia find impairments, the present study found enhanced electrophysiological effects in PSZ. Specifically, PSZ exhibited an unusually strong suppression of the P1 wave for unattended stimuli in the periphery when attending centrally and an unusually large P3b difference between rare and frequent stimuli in the center of gaze when attending to the periphery. These supranormal effects cannot be explained by a generalized deficit, by a failure to comprehend or maintain task instructions, by deficits in sensory processing, by lapses of attention, or by any other common explanation of impaired cognition in schizophrenia. In contrast, they were directly predicted by the hyperfocusing hypothesis.

Given that the present study examined chronic, medicated patients, there is always the possibility that the observed attentional differences were a side effect of antipsychotic medication use. However, medication dosage was uncorrelated with the effects, and there are no findings to suggest that the particular pattern of effects observed in the present study would be produced by antipsychotic medications.

\section{Underlying neural circuitry}

Although the ERP and behavioral data obtained in the present study cannot reveal the specific neural circuitry underlying hyperfocusing, the hyperfocusing hypothesis was inspired by computational neuroscience models of local cortical circuitry (Rolls et al., 2008; for review, see Durstewitz and Seamans, 2008). In these models, the $\mathrm{D}_{1}$ and $\mathrm{D}_{2}$ classes of dopamine receptors interact with NMDA- and GABAmediated processes to produce two competing attractor states: (1) a $\mathrm{D}_{1}$-dominated state with deep basins of attraction that leads to exaggerated winner-take-all dynamics and (2) a $\mathrm{D}_{2}$-dominated state with shallow basins of attraction that promotes flexibility and rapid updating of representations.

The known pathophysiology of schizophrenia is consistent with a disruption of these competing network states, and one possibility is that PSZ are biased toward the D1-dominated state that emphasizes winner-take-all processing (Durstewitz and Seamans, 2008). This winner-take-all processing could then manifest as hyperfocusing in a broad range of tasks. In the domain of spatial attention, it could explain both the present finding of hyperfocusing on the center of gaze and previous findings of larger spatial cuing effects and deficits in spreading attention broadly (Hahn et al., 2012a; Spencer et al., 2011; Gray et al., 2014). Exaggerated winner-take-all processing could also explain previous findings of reduced working memory capacity and a narrower span of attention in PSZ (Lee and Park, 2005; Piskulic et al., 2007; Elahipanah et al., 2010, 2011; Leonard et al., 2012; Johnson et al., 2013). Hyperfocusing on task goals could also explain classic findings of perseveration (Goldberg et al., 1987) and re- 
cent studies in which PSZ exhibited exaggerated distraction by stimuli that were task-irrelevant but partially matched task goals (Mayer et al., 2012; Luck et al., 2014; Sawaki et al., 2017). Thus, although hyperfocusing cannot explain the entirety of cognitive dysfunction in a complex disorder such as schizophrenia, it may provide a unified explanation of multiple disparate impairments.

\section{References}

Andreasen NC (1984) Scale for the assessment of negatives symptoms (SANS). Iowa City, IA: University of Iowa.

Andreasen NC, Pressler M, Nopoulos P, Miller D, Ho BC (2010) Antipsychotic dose equivalents and dose-years: a standardized method for comparing exposure to different drugs. Biol Psychiatry 67:255-262. CrossRef Medline

Ball K, Owsley C (1993) The useful field of view test: a new technique for evaluating age-related declines in visual function. J Am Optom Assoc 64:71-79. Medline

Ball K, Roenker DL (1998) UFOV useful field of view manual. San Antonio, TX: Psychological Corporation.

Brooks JL, Zoumpoulaki A, Bowman H (2017) Data-driven region-ofinterest selection without inflating Type I error rate. Psychophysiology 54:100-113. CrossRef Medline

Cramer AO, van Ravenzwaaij D, Matzke D, Steingroever H, Wetzels R, Grasman RP, Waldorp LJ, Wagenmakers EJ (2016) Hidden multiplicity in exploratory multiway ANOVA: prevalence and remedies. Psychon Bull Rev 23:640-647. Medline

Delorme A, Makeig S (2004) EEGLAB: an open source toolbox for analysis of single-trial EEG dynamics including independent component analysis. J Neurosci Methods 134:9-21. CrossRef Medline

Di Russo F, Martínez A, Hillyard SA (2003) Source analysis of event-related cortical activity during visuo-spatial attention. Cereb Cortex 13:486-499. CrossRef Medline

Durstewitz D, Seamans JK (2008) The dual-state theory of prefrontal cortex dopamine function with relevance to catechol-o-methyltransferase genotypes and schizophrenia. Biol Psychiatry 64:739-749. CrossRef Medline

Elahipanah A, Christensen BK, Reingold EM (2010) Visual search performance among persons with schizophrenia as a function of target eccentricity. Neuropsychology 24:192-198. CrossRef Medline

Elahipanah A, Christensen BK, Reingold EM (2011) Controlling the spotlight of attention: visual span size and flexibility in schizophrenia. Neuropsychologia 49:3370-3376. CrossRef Medline

First MB, Spitzer RL, Gibbon M, Williams JBW (1997) Structured Clinical Interview for DSM-IV- Axis I Disorders (SCID-I). Washington, DC: American Psychiatric.

Fischer B, Weber H (1993) Express saccades and visual attention. Behav Brain Sci 16:553-567. CrossRef

Goldberg TE, Weinberger DR, Berman KF, Pliskin NH, Podd MH (1987) Further evidence for dementia of the prefrontal type in schizophrenia? A controlled study of teaching the Wisconsin Card Sorting Test. Arch Gen Psychiatry 44:1008-1014. CrossRef Medline

Gray BE, Hahn B, Robinson B, Harvey A, Leonard CJ, Luck SJ, Gold JM (2014) Relationships between divided attention and working memory impairment in people with schizophrenia. Schizophr Bull 40:1462-1471. CrossRef Medline

Hahn B, Robinson BM, Harvey AN, Kaiser ST, Leonard CJ, Luck SJ, Gold JM (2012a) Visuospatial attention in schizophrenia: deficits in broad monitoring. J Abnorm Psychol 121:119-128. Medline

Hahn B, Hollingworth A, Robinson BM, Kaiser ST, Leonard CJ, Beck VM, Kappenman ES, Luck SJ, Gold JM (2012b) Control of working memory content in schizophrenia. Schizophr Res 134:70-75. CrossRef

Hawk AB, Carpenter WT Jr, Strauss JS (1975) Diagnostic criteria and fiveyear outcome in schizophrenia. A report from the International Pilot Study of schizophrenia. Arch Gen Psychiatry 32:343-347. Medline

Hillyard SA, Vogel EK, Luck SJ (1998) Sensory gain control (amplification) as a mechanism of selective attention: electrophysiological and neuroimaging evidence. Philos Trans R Soc Lond Biol Sci 353:1257-1270. CrossRef

Isreal JB, Chesney GL, Wickens CD, Donchin E (1980) P300 and tracking difficulty: Evidence for multiple resources in dual-task performance. Psychophysiology 17:259-273. CrossRef Medline
Jeon YW, Polich J (2003) Meta-analysis of P300 and schizophrenia: patients, paradigms, and practical implications. Psychophysiology 40:684701. CrossRef Medline

Johnson MK, McMahon RP, Robinson BM, Harvey AN, Hahn B, Leonard CJ, Luck SJ, Gold JM (2013) The relationship between working memory capacity and broad measures of cognitive ability in healthy adults and people with schizophrenia. Neuropsychology 27:220-229. CrossRef Medline

Jung TP, Makeig S, Westerfield M, Townsend J, Courchesne E, Sejnowski T] (2000) Removal of eye activity artifacts from visual event-related potentials in normal and clinical subjects. Clin Neurophysiol 111:1745-1758. CrossRef Medline

Kappenman ES, Luck SJ (2016) Best practices for event-related potential research in clinical populations. Biol Psychiatry Cogn Neurosci Neuroimaging 1:110-115. CrossRef Medline

Lavie N (1995) Perceptual load as a necessary condition for selective attention. J Exp Psychol Hum Percept Perform 21:451-468. CrossRef Medline

Lavie N (2010) Attention, distraction, and cognitive control under load. Curr Dir Psychol Sci 19:143-148. CrossRef

Lee J, Park S (2005) Working memory impairments in schizophrenia: a meta-analysis. J Abnorm Psychol 114:599-611. CrossRef Medline

Leonard CJ, Kaiser ST, Robinson BM, Kappenman ES, Hahn B, Gold JM, Luck SJ (2013) Toward the neural mechanisms of reduced working memory capacity in schizophrenia. Cereb Cortex 23:1582-1592. Medline

Leonard CJ, Robinson BM, Kaiser ST, Hahn B, McClenon C, Harvey AN, Luck SJ, Gold JM (2013) Testing sensory and cognitive explanations of the antisaccade deficit in schizophrenia. J Abnorm Psychol 122:11111120. CrossRef Medline

López V, López-Calderón J, Ortega R, Kreither J, Carrasco X, Rothhammer P, Rothhammer F, Rosas R, Aboitiz F (2006) Attention-deficit hyperactivity disorder involves differential cortical processing in a visual spatial attention paradigm. Clin Neurophysiol 117:2540-2548. CrossRef Medline

Lopez-Calderon J, Luck SJ (2014) ERPLAB: an open-source toolbox for the analysis of event-related potentials. Front Hum Neurosci 8:213. Medline

Luck SJ (1995) Multiple mechanisms of visual-spatial attention: Recent evidence from human electrophysiology. Behav Brain Res 71:113-123. CrossRef Medline

Luck SJ (2014) An introduction to the event-related potential technique, Ed 2. Cambridge, MA: MIT.

Luck SJ, Gaspelin N (2017) How to get statistically significant effects in any ERP experiment (and why you shouldn't). Psychophysiology 54:146157. CrossRef Medline

Luck SJ, Hillyard SA (1995) The role of attention in feature detection and conjunction discrimination: an electrophysiological analysis. Int J Neurosci 80:281-297. CrossRef Medline

Luck SJ, Hillyard SA, Mouloua M, Woldorff MG, Clark VP, Hawkins HL (1994) Effects of spatial cuing on luminance detectability: psychophysical and electrophysiological evidence for early selection. J Exp Psychol Hum Percept Perform 20:887-904. CrossRef Medline

Luck SJ, Kappenman ES, Fuller RL, Robinson B, Summerfelt A, Gold JM (2009) Impaired response selection in schizophrenia: evidence from the P3 wave and the lateralized readiness potential. Psychophysiology 46: 776-786. CrossRef Medline

Luck SJ, McClenon C, Beck VM, Hollingworth A, Leonard CJ, Hahn B, Robinson BM, Gold JM (2014) Hyperfocusing in schizophrenia: Evidence from interactions between working memory and eye movements. J Abnorm Psychol 123:783-795. CrossRef Medline

Mayer JS, Fukuda K, Vogel EK, Park S (2012) Impaired contingent attentional capture predicts reduced working memory capacity in schizophrenia. PLoS One 7:e48586. CrossRef Medline

Myers RS, Ball KK, Kalina TD, Roth DL, Goode KT (2000) Relation of useful field of view and other screening tests to on-road driving performance. Percept Motor Skills 91:279-290. CrossRef Medline

Nuechterlein KH, Green MF, Kern RS, Baade LE, Barch DM, Cohen JD, Essock S, Fenton WS, Frese III PD, Frederick J, Gold JM (2008) The MATRICS Consensus Cognitive Battery, part 1: test selection, reliability, and validity. Am J Psychiatry 165:203-213. Medline

Overall JE, Gorham DR (1962) The brief psychiatric rating scale. Psychol Rep 10:799-812. CrossRef

Piskulic D, Olver JS, Norman TR, Maruff P (2007) Behavioural studies of spatial working memory dysfunction in schizophrenia: a quantitative literature review. Psychiatry Res 150:111-121. CrossRef Medline 
Polich J (2012) Neuropsychology of P300. In: Oxford handbook of eventrelated potential components (Luck SJ, Kappenman ES, eds), pp. 159188. New York: Oxford University Press.

Potts GF, O’Donnell BF, Hirayasu Y, McCarley RW (2002) Disruption of neural systems of visual attention in schizophrenia. Arch Gen Psychiatry 59:418-424. CrossRef Medline

Rolls ET, Loh M, Deco G, Winterer G (2008) Computational models of schizophrenia and dopamine modulation in the prefrontal cortex. Nat Rev Neurosci 9:696-709. CrossRef Medline

Sawaki R, Geng JJ, Luck SJ (2012) A common neural mechanism for preventing and terminating the allocation of attention. J Neurosci 32:1072510736. CrossRef Medline
Sawaki R, Kreither J, Leonard CJ, Kaiser ST, Hahn B, Gold JM, Luck SJ (2017) Hyperfocusing on goal-related information in schizophrenia: Evidence from electrophysiology. J Abnorm Psychol 126:106-116. CrossRef Medline

Spencer KM, Nestor PG, Valdman O, Niznikiewicz MA, Shenton ME, McCarley RW (2011) Enhanced facilitation of spatial attention in schizophrenia. Neuropsychology 25:76-85. CrossRef Medline

Wechsler D (1999) Wechsler Abrbreviated Scale of Intelligence (WASI). San Antonio, TX: Harcourt Asessment.

Woodman GF, Luck SJ (2003) Serial deployment of attention during visual search. J Exp Psychol Hum Percept Perform 29:121-138. CrossRef Medline 